\title{
The Significance of Strengthening the Study of Molecular Imaging and the Way of Learning for Radiologists
}

\author{
Ge Yan-ming, Tu Ying-shan, Zhu Hong, Long Jin-feng, Wang Xi-zhen, Dong Peng* \\ Medical Imaging Center of the Affiliated Hospital \\ Weifang Medical University \\ Weifang 261041 P. R. China
}

\begin{abstract}
Medical molecular biology is developing rapidly in recent years, whose theories and techniques have promoted the development of medical imaging, and it made huge influence on the modern medical imaging. Medical molecular biology's development has extremely improved people's understanding and treatment on the diseases. The development of molecular imaging was based on the development of medical molecular biology, which is the most important branch of medical imaging. So, it is necessary and urgent for the radiologists to master the basic knowledge and new developments of medical molecular biology. This paper aimed to discuss the significance and ways of learning the medical molecular biology.
\end{abstract}

Keywords-Learn; Medical molecular biology; Medical imaging

\section{INTRODUCTION}

The rapid development of modern medicine requires the physician to work hard and learn more knowledge. It is reported that the development of medical molecular biology further enhanced the clinicians' awareness of the diseases. The research of modern molecular biology refers to the nature of diseases, which affected the development of modern medicine especially the medical imaging [1,2]. In 21th century, it has become an important way to study the occurrence, development and treatment of the lesions in the molecular and cellular levels. Recently, medical molecular biology's methods and technologies have been used widely in the basic and clinical studies, which lead to the newly developing discipline-molecular imaging, and it is the hot spot in the studies of medical imaging [3, 4]. It is very important for the radiologists to learn and use medical molecular biology's knowledge, such as helping radiologists to know the latest information and hot spot of diseases in the molecular and cellular levels, improving clinical diagnostic ability and level, etc. However, because of the lack of relevant knowledge or outdated knowledge, some radiologists are limited to diagnosing diseases, evaluating curative effect and judging prognosis of the patients especially in the diagnosis by using molecular imaging. So, with the emergence of molecular imaging and the development of medical molecular biology, radiologists are required to master the related theories and

This work was supported by a grant from the Foundation of Chinese Medical Association Medical Education Branch (2016B-YJS031), Foundation of Higher medical education research center of Shandong Province

(YJKT201514), Foundation of Postgraduate education innovation program of Shandong Province (SDYY15119). Corresponding author: DONG Peng, Email: dongpeng98021@sina.com skills of the medical molecular biology, and then they could do efficiently in clinical work and scientific researches about the medical imaging.

\section{THE VALUE OF MEDICAL MOLECULAR BIOLOGY FOR THE MEDICAL IMAGING}

In the latest years, medical molecular biology developed rapidly in its theory and application, which has permeated through the medical areas. Molecular imaging which is a newly emerging discipline is at the forefront of the development of medical imaging. The emergency and development of molecular imaging will lead the direction of the medical imaging [5]. Different from the morphological description of the traditional radiology, molecular imaging applies medical imaging devices to observe non-invasively the physiological and pathological process of lesions by the means of functional magnetic resonance imaging, molecular probe and contrast material, etc. It can make qualitative or quantitative observation of the pathological changes in the molecular and cellular levels before organs or tissues changing their forms [6]

For example, magnetic resonance spectrum can show tumor's metabolism and response to the therapy. According to marking the specific factors of the lesions, MRI can show the pathological and biological changes when lesions happen and develop, and molecular imaging helps to diagnose some type tumors in early stage and to do researches on the mechanism of lesions. With the help of computed tomography energy spectrum imaging or the molecular probe, computed tomography examination or magnetic resonance imaging can determine the nature of tumors and their metastasis in some extent. So, radiologists can make qualitative diagnosis and judge the stage of the lesions. In the study of treatment effect of the lesions, based on the markers of the lesions or the magnetic resonance spectrum technique, the changing of the masses in the shape and volume can be shown directly, and the metabolism of the masses may be shown, which gives obvious reference to the judgment of treatment effect. The techniques of molecular imaging not only provide significant ways for related fundamental researches but play an crucial role in clinical study [7].

In the newly developing medical model, molecular imaging techniques may help to treat diseases while diagnosing in the 
early stage and trace the changes after treatment so that link diagnosis with treatment.

\section{THE NECESSITY FOR THE RADIOLOGISTS TO MASTER THE KNOWLEDGE OF MEDICAL MOLECULAR BIOLOGY}

The molecular imaging is a newly emerging discipline based on the development of the medical molecular biology, computed science and medical imaging, etc. Molecular imaging uses some available medical imaging techniques, such as nuclear medicine and magnetic resonance imaging, to take images lively in the physiological and pathological processes at the molecular level inside human body. It's a safe technique which can be repeated and have immeasurable functions in diagnosis, treatment and assessment of the clinical treatment and mechanism.

As a radiologist who want to master and use the molecular imaging, he should learn not only the conventional radiological knowledge but the knowledge of the medical molecular biology, such as the mechanism and techniques of molecular probe, how to choose the specific factor of the tumors, the analysis shown in related imaging, etc. The molecular imaging changes from simple morphological study to functional analysis, from subjective diagnosis into qualitative and quantitative analysis. So, radiologists must learn the knowledge of medical molecular biology, and make progresses in inventing molecular probe, specific marker and the performance of molecular imaging. They should show the occurrence and the development of diseases directly in early stage and the effect after treatment by the means of advanced imaging. So, they can achieve the long-term development of molecular imaging. Because of the emergency of related techniques, molecular imaging places more emphasis on the analysis of individual difference, which give important opportunity for the individual diagnosis-accurate imaging diagnosis.

In the future, the molecular imaging is to proceed with the developing process of individual accurate imaging diagnosis, such as picking out specific diagnostic markers of tumor, making corresponding specific molecular probe, making fittest diagnostic plan for patients by the individual analysis of molecular imaging, and providing with the functions of supervision and evaluation, so that realize the union of diagnosis and treatment. In a word, it has enormous leading significance in improving radiologists' comprehensive diagnostic and curative ability by mastering the knowledge of medical molecular biology. Currently, many medical universities in China have established courses about medical molecular biology and related experiences; they also have corresponding planning and experimental materials. So, radiologists who graduate from medical university know the basic knowledge of medical molecular biology. However, because the theories and techniques of medical molecular biology update continually, radiologists have to keep studying personally so that they will know the latest development about medical molecular biology. The medical molecular biology is a new area which has no professional physicians, and we need to study again [8].

\section{THE WAY TO STRENGTHEN THE LEARNING OF MEDICAL MOLECULAR BIOLOGY}

Radiologists should realize the importance of learning knowledge of medical molecular biology and study positively. Except for the study arranged by hospitals, department and disciplinary team, more importantly, they should learn by themselves and get necessary related theories and skills through continuing education. In the process of continuing education, radiologists need control the depth and width of study for themselves. Practical work need the research of the mechanism of diseases, the evaluation of treatment and the judgment of prognosis, which requires radiologists to master the related theories and related skills of the medical molecular biology, then they can adapt better to the clinical practice.

There are so many ways for radiologists to get medical molecular biology's knowledge: (1) Learning the basic theory of medical molecular biology roundly and systematically. The radiologists need to choose reference books according to their own condition. They can give priority to country planning teaching materials so that they can master the theories gradually and understand different kinds of frequent-used nouns, terms and some involved research areas. Recently, the medical public class established by medical universities on the Internet is becoming more and more complete and perfect. Student can also learn by massive open online courses, which helps to understand and master the knowledge [9]. Know the latest development and research trends through professional magazine and document. (2) Determine the direction and learn the related professional skills. The study of molecular imaging refers to many disciplines, so radiologists should determine their own research direction and learn something targeted in study.

It is easy and convenient to study on the Internet, its comprehensive contents and convenient search play an irreplaceable role in medical continuing education. We can use nets, such as CNKI (China national knowledge infrastructure) to choose documents and resource to study. In addition, we can not only follow the latest trends but communicate and discuss with others through other professional nets of medical molecular biology. Emphasize the academic exchange and cooperative research. It is essential to participate in lectures or special meetings, especially the national or international activities. We can know the latest applications and trends of molecular imaging and medical molecular biology through this academic communication. Furthermore, we can discuss directly with our colleagues or experts so that we can get necessary indication and help.

Nowadays, with the fast development of science and technique, the molecular imaging can't be developed only by radiologists. Only the cooperation with the experts of medical molecular biology can promote the development of molecular imaging and its clinical applications. As a new subject, the development of the molecular imaging needs everyone's effort, and radiologists should take the responsibility of promoting the developing of the molecular imaging. The radiologists should not only communicate and work with the fundamental disciplines but make the best use of bilateral advantages. So, there is no doubt that strengthening the cooperation and 
communication helps to deal with the problems involved in the development of molecular imaging and promote radiologists' research and study of medical molecular biology.

\section{SUMMARY}

In brief, medical molecular biology is a powerful medical leading discipline. The emergency of molecular imaging is the result of medical development caused by the medical molecular imaging's theories and techniques. As the radiologists of 21th century, they must pay high attention to the learning and researching of the molecular imaging. Only when they learn and use the related fundamental knowledge and techniques well, they can adapt to the medical imaging development in 21th century and provide the clinical work with better service.

\section{ACKNOWLEDGMENT}

This work was supported by a grant from the Foundation of Chinese Medical Association Medical Education Branch (2016B-YJS031), Foundation of Higher medical education research center of Shandong Province (YJKT201514), Foundation of Postgraduate education innovation program of Shandong Province (SDYY15119).

\section{REFERENCES}

[1] Feng Z. Medical molecular biology. People's Medical Publishing House,Beijing,2001.

[2] Fang FD. Developing History and Prospecting of Medical Molecular Biology. Medicine and Philosophy 1999, 20(1):17-20.

[3] Zhang LJ, Song GY, Bao YM. The research and development of molecular imaging. Chin J Radiol 2002, 36(10):950-953.

[4] Dong P, Wang B, Sun YQ, et al. Analysis of the relationship between the construction of molecular imaging and the cultivation of innovative ability of Graduate student in medical imaging [J].China Higher Medical Education 2008, (6):117-118.

[5] Shen BZ. Unlimited potential Charm-Review and prospect of molecular imaging research. Chin J Radiol 2014, (5):353-357.

[6] Perrone A. Molecular imaging technologies and translational medicine. The Journal of Nuclear Medicine 2008, 49(12):25N.

[7] Shen BZ, Wang W. 2011 Annual Report on molecular imaging. China Continuing Medical Education 2011, (8):132-166.

[8] Zhang P, Wang YS. Strengthening continuing education of medical molecular biology and enhancing ophthalmologists' overall diagnosis and treatment level. Northwest Medical Education 2009, 17(5):10261028.

[9] Yu Q, He PF,Yuan YX, et al. Development of MOOC and its enlightenmengs on higher medical education. Chin J Med Libr Inf Sci 2014, 23(7):8-12.

[10] WEN Ming, LI Shao-lin. Application of MRI in molecular imaging. C hin J M ed Imagin g Technol 2007, 23(1):147-150. ( In Chinese)

[11] TENG Gao-jun, CUI Ying. Research progress in molecular magnetic resonance imaging. Chin J Magn Reson Imaging, 2014, 5:31-36. ( In Chinese)

[12] ZHANG Gui-xiang, ZHAO Jing-long, LI Yu-jie, et al. Research category and progress of molecular imaging. Chin J Med Imaging Technol 2005, 21(11):1636-1639. ( In Chinese)

[13] LIU Guang-yuan, LIU Xin-yao. A research of present research and development of molecular imaging homeand abroad. J Med Postgra 2010,23(3):323-326.( In Chinese)

[14] ZHU Hai- yun, CHENG Yong-de, SHEN Bao-zhong. Recent research progress in interventional molecular imaging. J Intervent Radiol 2016 25(1):1-6. ( In Chinese)

[15] TANG Hui, YU Lu, WANG Qi,et al. Discussion on the comprehensive qualities of Radiological Technicians in modern medical imaging technology. Continuing medical education 2016,30(2):3. ( In Chinese)

[16] JIN Zheng-yu, XUE Hua-dan. Status Quo and Prospect of Clinical Molecular Imaging. ACTA ACADEMIAE MEDICINAE SINICAE 2009, 31(2):121-123. 